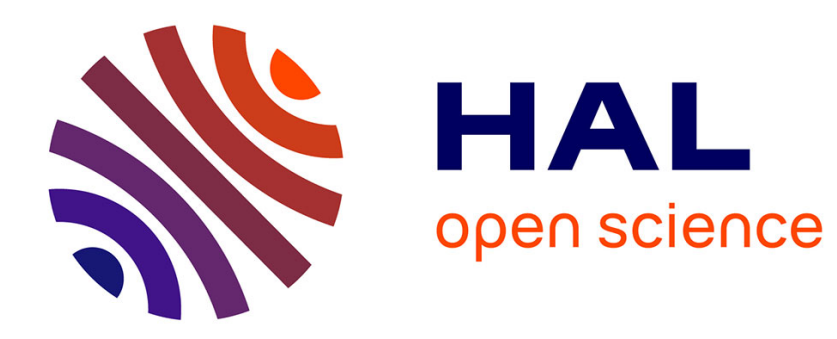

\title{
Fano manifolds of Calabi-Yau Hodge type
}

Atanas Iliev, Laurent Manivel

\section{To cite this version:}

Atanas Iliev, Laurent Manivel. Fano manifolds of Calabi-Yau Hodge type. Journal of Pure and Applied Algebra, 2015, 219 (6), pp.2225-2244. hal-00566547

\section{HAL Id: hal-00566547 https://hal.science/hal-00566547}

Submitted on 16 Feb 2011

HAL is a multi-disciplinary open access archive for the deposit and dissemination of scientific research documents, whether they are published or not. The documents may come from teaching and research institutions in France or abroad, or from public or private research centers.
L'archive ouverte pluridisciplinaire $\mathbf{H A L}$, est destinée au dépôt et à la diffusion de documents scientifiques de niveau recherche, publiés ou non, émanant des établissements d'enseignement et de recherche français ou étrangers, des laboratoires publics ou privés. 


\title{
FANO MANIFOLDS OF CALABI-YAU TYPE
}

\author{
ATANAS ILIEV AND LAURENT MANIVEL
}

\begin{abstract}
We introduce and we study a class of odd dimensional compact complex manifolds whose Hodge structure in middle dimension looks like that of a Calabi-Yau threefold. We construct several series of interesting examples from rational homogeneous spaces with special properties.
\end{abstract}

\section{INTRODUCTION}

If we think, quite naively, to mirror symmetry as a kind of mysterious process exchanging families of Calabi-Yau threefolds in such a way that the Hodge diamond rotates by a quarter-turn, an obvious difficulty arises for rigid Calabi-Yau's: their mirrors could not be Kähler! The solution given to that issue in $\mathrm{CDP}$, Sch was to describe the mirror of certain rigid Calabi-Yau threefolds as families of higher dimensional manifolds of a very special kind. In particular, these mirror manifolds have odd dimension $2 n+1$ and their Hodge structure in middle dimension looks like that of a Calabi-Yau threefold, in the sense that the only non-zero Hodge numbers are $h^{n-1, n+2}=h^{n+2, n-1}=1$ and $h^{n, n+1}=h^{n+1, n}$.

This is precisely the kind of manifolds that we study in this paper, with the idea that, under some conditions that will be made more precise below, these manifolds of Calabi-Yau type should share some of the very nice properties of Calabi-Yau threefolds. The definition that we will use may not exactly be the correct one and we consider it as provisional. Our main general result will be that for a manifold of Calabi-Yau type with non-obstructed deformations, it implies that the relative intermediate Jacobian forms an integrable system over the gauged moduli space - a result due to Donagi and Markman for Calabi-Yau threefolds. The period map also has a very similar behavior to the Calabi-Yau setting. Related homological properties are explored in [IK], where it is proved that one can extend at least to certain Fano manifolds of Calabi-Yau type, the celebrated result of Voisin according to which the Griffiths group of a Calabi-Yau threefold cannot be finitely generated.

But the main goal of the present paper is to construct examples of manifolds of Calabi-Yau type, which will all be Fano's. Our main source of examples will be complete intersections in homogeneous spaces: we will show that, for such a construction to work, one needs strong numerical coincidences, notably between the dimension and the index of the ambient 
homogeneous space. These coincidences are observed in some cases. Notably, we construct interesting examples as quadratic sections of homogeneous spaces that are Mukai varieties of even dimension (Proposition 3.3), while for Mukai varieties of odd dimension we need to take double covers branched over quadratic sections (Proposition 3.6).

But our most intriguing series of examples is constructed from homogeneous spaces with the property that their projective dual is a hypersurface of degree equal to the coindex minus one (the coindex of a Fano manifold being defined as the dimension minus the index). We are aware of four cases for which this strange coincidence can be observed, and we show that suitable linear sections provide examples of Fano manifolds with the required Hodge numbers. In fact we can really conclude that they are of Calabi-Yau type only in half of the cases; for the two others there remains a tedious computation to be done, but we have no doubt about the fact that the final conclusion should be positive. In each case, we would conclude that a certain type of hypersurfaces can be, generically, represented as a linear section of the dual hypersurface to our homogeneous space, in a finite number of ways. For example, a generic cubic sevenfold can be represented as a linear section of the famous Cartan cubic, the $E_{6}$-invariant hypersurface in $\mathbb{P}^{26}$, in a finite number of different ways. This has interesting consequences for its derived category, as we show in [IM2] where this example in studied in more details.

It would be interesting to find out more examples of Fano manifolds of Calabi-Yau type. A possible strategy would be to start from rigid CalabiYau threefolds and try to identify their mirrors systematically. We expect that these varieties will exhibit a rich and interesting geometry.

\section{Definition AND FIRST PROPERTIES}

We start with our main definition.

Definition 2.1. Let $X$ be a smooth complex compact variety of odd dimension $2 n+1, n \geq 1$. We call $X$ a manifold of Calabi-Yau type if

(1) The middle dimensional Hodge structure is numerically similar to that of a Calabi-Yau threefold, that is

$$
h^{n+2, n-1}(X)=1, \quad \text { and } \quad h^{n+p+1, n-p}(X)=0 \text { for } p \geq 2 .
$$

(2) For any generator $\omega \in H^{n+2, n-1}(X)$, the contraction map

$$
H^{1}(X, T X) \stackrel{\omega}{\longrightarrow} H^{n-1}\left(X, \Omega_{X}^{n+1}\right)
$$

is an isomorphism.

(3) The Hodge numbers $h^{k, 0}(X)=0$ for $1 \leq k \leq 2 n$.

A Calabi-Yau threefold is of course a manifold of Calabi-Yau type. The definition may not be the optimal one, since in particular, even in dimension three, there exist manifolds of Calabi-Yau type which are not Calabi-Yau stricto sensu. Also, the condition of being a manifold of Calabi-Yau type is 
clearly invariant under small deformations but probably not under arbitrary deformations, contrary to being Calabi-Yau.

Rather, our main motivation is to find interesting examples of manifolds of Calabi-Yau type, and to investigate their geometry. In many respects they will behave like Calabi-Yau threefolds, and sometimes the examples we will meet will be related, in a rather non trivial way, to Calabi-Yau threefolds. Sometimes we will observe interesting differences of behavior.

Deformations of Calabi-Yau manifolds are always unobstructed, as follows from the celebrated Tian-Todorov theorem. We don't know whether this always remains true for manifolds of Calabi-Yau type. (Actually all the concrete examples that we deal with are Fano, hence have unobstructed deformations.) Nevertheless, our first observation is that versal deformations of manifolds of Calabi-Yau type give rise, exactly as families of Calabi-Yau threefolds do, to some beautiful integrable systems.

Consider a versal family $\pi: \mathcal{X} \rightarrow \mathcal{B}$ of manifolds of Calabi-Yau type, with special fiber $X$. We suppose that the base $\mathcal{B}$ is identified with an open subset of $H^{1}(X, T X)$. Since $h^{n+p+1, n-p}(X)=0$ for $p \geq 2$, the line bundle $R^{n-1} \pi_{*} \Omega_{\mathcal{X} / \mathcal{B}}^{n+2}$ over $\mathcal{B}$ is holomorphic, and the complement $\mathcal{B}_{*}$ of the zero section, with the pull-back $\mathcal{X}_{*}$ of $\mathcal{X}$, defines the family of gauged manifolds of Calabi-Yau type.

Recall that the intermediate Jacobian of $X$ is the complex torus

$$
J(X)=F^{n+1} H^{2 n+1}(X, \mathbb{C})^{\vee} / H_{2 n+1}(X, \mathbb{Z}) .
$$

Since $X$ is of Calabi-Yau type, the dimension of $J(X)$ is $h^{n+1, n}(X)+1=$ $h^{1}(T X)+1=\operatorname{dim} \mathcal{B}_{*}$. Globalizing the construction, we get a torus bundle, the relative intermediate Jacobian

$$
\mathcal{J}(\mathcal{X} / \mathcal{B}) \longrightarrow \mathcal{B}_{*}
$$

The theorem proved by Donagi and Markman for Calabi-Yau threefolds [DM] can be extended to our generalized setting:

Theorem 2.2. The torus fibration $\mathcal{J}(\mathcal{X} / \mathcal{B}) \longrightarrow \mathcal{B}_{*}$ defines a completely integrable Hamiltonian system.

Proof. The proof of the Theorem for Calabi-Yau threefolds is easy to extend to our setting. The main observation is that the tangent exact sequence of the $\mathbb{C}^{*}$-bundle $\mathcal{B}_{*} \rightarrow \mathcal{B}$ can be identified with the Hodge filtration of $H^{2 n+1}\left(\mathcal{X}_{*} / \mathcal{B}_{*}, \mathbb{C}\right)$. More precisely there is a commutative diagram

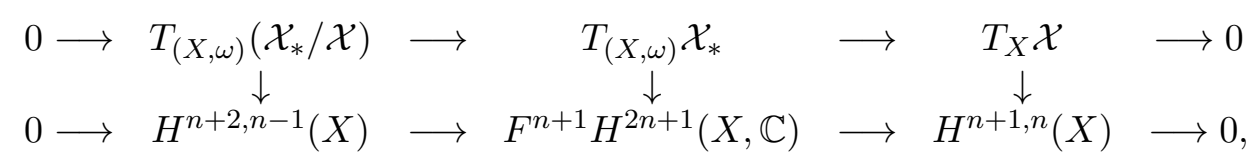

where the vertical maps are all isomorphisms. In particular the middle one is given by the differential of $\omega$ (considered as a section on $\mathcal{X}_{*}$ of the holomorphic bundle $\left.F^{n+2} H^{2 n+1}\left(\mathcal{X}_{*} / \mathcal{B}_{*}, \mathbb{C}\right)\right)$, by the Gauss-Manin connection: 
this yields, by Griffiths' transversality, a map

$$
\nabla \omega: T_{(X, \omega)} \mathcal{X}_{*} \longrightarrow F^{n+1} H^{2 n+1}(X, \mathbb{C}),
$$

which must be an isomorphism since its two graded parts are.

This observation yields a global identification between the cotangent bundle of $\mathcal{X}_{*}$ and the dual of the Hodge bundle $F^{n+1} H^{2 n+1}\left(\mathcal{X}_{*} / \mathcal{B}_{*}, \mathbb{C}\right)$. Now $\Omega_{\mathcal{X}}$ has a canonical symplectic structure. We need to check that it descends to the torus fibration $\mathcal{J}(\mathcal{X} / \mathcal{B})$, which is just a quotient by the relative lattice defined by locally constant $(n+1)$-cycles. So there remains to verify that such a locally constant cycle, say $\gamma$, when considered as a one-form on $\mathcal{X}_{*}$, is closed. But the argument of Donagi-Markman, which consists in constructing a local primitive by integrating $\omega$ over $\gamma$, adapts mutatis mutandis.

Remark. This implies the existence of a special Kähler structure on the base, with an extremely rich geometry $[\mathrm{Fr}$.

The existence of that integrable system is related to that of a certain cubic form on the base manifold, which in the usual Calabi-Yau setting is the famous Yukawa cubic. The Yukawa cubic is defined, for a gauged Calabi-Yau threefold $(X, \omega)$, by the composition

$$
\operatorname{Sym}^{3} H^{1}(X, T X) \longrightarrow H^{3}\left(X, \omega_{X}^{-1}\right) \stackrel{S D}{\longrightarrow} H^{0}\left(X, \omega_{X}^{2}\right)^{\vee} \stackrel{\omega^{2}}{\longrightarrow} \mathbb{C},
$$

where $S D$ denotes Serre duality. In our generalized setting, this construction can be extended as follows. First consider the natural map $\wedge^{n-1} T X \otimes$ $\wedge^{n-1} T X \rightarrow \wedge^{2 n-2} T X$, which is symmetric if $n$ is odd and skew-symmetric if $n$ is even. Twisting by $\omega_{X}^{2}$ we get a map $\Omega_{X}^{n+2} \otimes \Omega_{X}^{n+2} \rightarrow \Omega_{X}^{3} \otimes \omega_{X}$ with the same parity. We get an induced map (always symmetric, since the cup-product has the same parity as the degree, here $n-1$ )

$$
\operatorname{Sym}^{2} H^{n-1}\left(X, \Omega_{X}^{n+2}\right) \longrightarrow H^{2 n-2}\left(X, \Omega_{X}^{3} \otimes \omega_{X}\right),
$$

sending the square of a generator $\omega$ of $H^{n-1}\left(X, \Omega_{X}^{n+2}\right)$ to an element $s(\omega)$ of $H^{2 n-2}\left(X, \Omega_{X}^{3} \otimes \omega_{X}\right)$. We can then define a map

$$
\operatorname{Sym}^{3} H^{1}(X, T X) \longrightarrow H^{3}\left(X, \wedge^{3} T X\right) \stackrel{s(\omega)}{\longrightarrow} H^{2 n+1}\left(X, \omega_{X}\right)=\mathbb{C},
$$

which is our generalized cubic at the point of $\mathcal{B}_{*}$ defined by the gauged manifold of Calabi-Yau type $(X, \omega)$.

For a slightly different view-point, consider the period map

$$
\mathfrak{P}: \begin{aligned}
\mathcal{B}_{*} & \rightarrow H^{2 n+1}\left(X_{0}, \mathbb{C}\right), \\
(X, \omega) & \mapsto \omega,
\end{aligned}
$$

defined once the family $\mathcal{X} \rightarrow \mathcal{B}$ has been trivialized (in the differentiable category). The same proof as in the usual Calabi-Yau setting leads to the following statement: 
Proposition 2.3. The period map of a versal family of manifolds of CalabiYau type is a local immersion.

Moreover, its image is a pointed cone in $H^{2 n+1}\left(X_{0}, \mathbb{C}\right)$, which is Lagrangian relatively to the symplectic structure defined by the intersection product.

It would be interesting to investigate under which conditions a Frobenius structure can be defined from our generalized Yukawa cubic. We know from [CDP that everything works fine for one of the most interesting examples of manifolds of Calabi-Yau type, the cubic sevenfold. Is there any chance to extend this to other examples? One can make a first step in this direction by mimicking the proof of Proposition 3.3 in $[\mathrm{VD}$, which allows one to prove that:

Proposition 2.4. One can choose a local section $\omega$ of $F^{n+2} H^{2 n+1}\left(\mathcal{X}_{*} / \mathcal{B}_{*}, \mathbb{C}\right)$ in such a way that the Yukawa cubic

$$
Y^{\omega}: \operatorname{Sym}^{3} H^{1}(X, T X) \longrightarrow H^{3}\left(X, \wedge^{3} T X\right) \stackrel{s(\omega)}{\longrightarrow} \mathbb{C}
$$

is defined by the derivatives of some potential $F$ on $\mathcal{B}$.

Understanding under which conditions this potential would satisfy the WDVV equation seems to be a very difficult problem, but the question is posed: could there be a version of mirror symmetry for (some) manifolds of Calabi-Yau type?

\section{Complete intersections of Calabi-Yau type}

3.1. Hodge numbers of complete intersections. In this section we review well-known results about the cohomology of complete intersections in weighted projective spaces. Let us begin with a degree $d$ hypersurface $X_{d} \subset w \mathbb{P}^{n}$, where $w=\left(w_{0}, \ldots, w_{n}\right)$ is the set of weights defining the weighted projective space $w \mathbb{P}^{n}$.

The usual projective space $\mathbb{P}^{n}$ corresponds to $w=(1, \ldots, 1)$. In that case, it is well known that the primitive cohomology of $X_{d}$ can be expressed in terms of the Jacobian ring

$$
R=\mathbb{C}\left[x_{0}, \ldots, x_{n}\right] /\left(\frac{\partial F}{\partial x_{0}}, \ldots \frac{\partial F}{\partial x_{n}}\right),
$$

where $F$ denotes an equation of $X_{d}$. In fact there exists a general relation of that kind for any quasi-smooth hypersurface $X_{d} \subset w \mathbb{P}^{n}$, which is due to Steenbrink $[\mathrm{St}]$ :

$$
H^{n-p-1, p}\left(X_{d}\right)_{0} \simeq R_{(p+1) d-|w|},
$$

where $|w|=w_{0}+\cdots+w_{n}$ and $R_{k}$ denotes the degree $k$ component of $R$, with respect to the natural grading defined by $\operatorname{deg}\left(x_{i}\right)=w_{i}$. 
An obvious consequence is that if $d$ divides $|w|$, that is, $|w|=(p+1) d$ form some $p$, then

$$
\begin{gathered}
H^{n-q-1, q}\left(X_{d}\right)_{0}=0 \quad \text { for } q \leq p-1, \\
H^{n-p-1, p}\left(X_{d}\right)_{0} \simeq R_{0} \simeq \mathbb{C}, \\
H^{n-p-2, p+1}\left(X_{d}\right)_{0} \simeq R_{d} \simeq H^{1}\left(X_{d}, T X_{d}\right) .
\end{gathered}
$$

In particular, if $n=2 m$ is even and $|w|=(m-1) d$, the primitive cohomology of $X_{d}$ defines a Hodge structure of the same type as that of a Calabi-Yau threefold. We find a short list of smooth examples in dimension $n-1 \geq 4$ :

$$
\begin{array}{ccccl}
n-1 & w & d & \text { moduli } & X \\
7 & 1^{9} & 3 & 84 & \text { cubic sevenfold } \\
5 & 1^{7} & 2,3 & 83 & \text { cubic section of } \mathbb{Q}^{6} \\
5 & 1^{6}, 2 & 4 & 90 & \text { double quartic fivefold }
\end{array}
$$

We will meet again the cubic sevenfolds later on. They will be investigated in a more systematic way in [IM2].

3.2. A technical lemma. We would like to generalize the previous examples and show that certain low degree hypersurfaces in homogeneous spaces are of Calabi-Yau type.

The general set-up would be the following. Suppose that $\Sigma$ is a Fano variety of dimension $d_{\Sigma}$, with $-K_{\Sigma}=\iota L$ for some ample line bundle $L$. Suppose moreover that $L$ is generated by global sections and consider the zero-locus $X$ of a general section of $L$. Its dimension is of course $d_{X}=$ $d_{\Sigma}-1$, and $-K_{X}=(\iota-1) L_{\mid X}$. The proof of our next technical lemma is a rather standard play with long exact sequences. It is essentially an application of Griffiths' techniques, for the computation of Hodge structures of hypersurfaces $\mathrm{Gr}, \mathrm{Vo}$.

Lemma 3.1. Let $\Sigma$ be such that $d_{\Sigma}=2 \iota+2$.

(1) Suppose that for $0 \leq k<p<\iota$,

$$
H^{d_{\Sigma}-p+k-1}\left(\Sigma, \Omega_{\Sigma}^{p-k} \otimes L^{-k}\right)=H^{d_{\Sigma}-p+k}\left(\Sigma, \Omega_{\Sigma}^{p-k} \otimes L^{-k-1}\right)=0 .
$$

Then $h^{p, d_{X}-p}(X)=0$ for $p<\iota$.

(2) Suppose that for $1 \leq k<\iota$ and $\epsilon, \epsilon^{\prime} \in\{0,1\}$,

$$
H^{\iota+k+1+\epsilon+\epsilon^{\prime}}\left(\Sigma, \Omega_{\Sigma}^{i-k} \otimes L^{-k+1-\epsilon}\right)=0 .
$$

Then $h^{\iota+2, \iota-1}(X)=1$.

(3) Suppose moreover that for $2 \leq k \leq \iota$ and $\epsilon, \epsilon^{\prime} \in\{0,1\}$,

$$
H^{\iota+k+1+\epsilon+\epsilon^{\prime}}\left(\Sigma, \Omega_{\Sigma}^{i-k} \otimes L^{-k-\epsilon}\right)=0 .
$$

Then $X$ is a manifold of Calabi-Yau type. 
Proof. Use the conormal sequence of the pair $(X, \Sigma)$. Its $p$-th wedge power is the complex

$$
0 \rightarrow L_{\mid X}^{-p} \rightarrow \Omega_{\Sigma}^{1} \otimes L_{\mid X}^{-p+1} \rightarrow \cdots \rightarrow \Omega_{\Sigma}^{p-1} \otimes L_{\mid X} \rightarrow \Omega_{\Sigma \mid X}^{p} \rightarrow \Omega_{X}^{p} \rightarrow 0 .
$$

Under hypothesis (1), for $p \leq \iota-2$, we have

$$
H^{d_{X}-p+k}\left(X, \Omega_{\Sigma}^{p-k} \otimes L_{\mid X}^{-k}\right)=0
$$

for all $k$ from 0 to $p$. Our first claim follows.

For $p=\iota-1$, our hypothesis imply that

$$
H^{d_{X}-p+k+\epsilon}\left(X, \Omega_{\Sigma}^{p-k} \otimes L_{\mid X}^{-k}\right)=0
$$

for $\epsilon \in\{0,1\}$. But then, it follows that

$$
H^{\iota+2}\left(X, \Omega_{X}^{\iota-1}\right)=H^{d_{X}}\left(X, L_{\mid X}^{-\iota+1}\right)=H^{d_{X}}\left(X, K_{X}\right)=\mathbb{C},
$$

which was our second claim.

Finally, for $p=\iota$ the hypothesis we made are such that we get a complex

$$
0 \rightarrow H^{\iota+1}\left(X, \Omega_{X}^{\iota}\right) \rightarrow H^{d_{X}}\left(X, L_{\mid X}^{-\iota}\right) \rightarrow H^{d_{X}}\left(X, \Omega_{\Sigma}^{1} \otimes L_{\mid X}^{-\iota+1}\right) .
$$

Using Serre duality, we get the dual complex

$$
H^{0}\left(X, T \Sigma_{\mid X}\right) \rightarrow H^{0}(X, L) \rightarrow H^{\iota}\left(X, \Omega_{X}^{\iota+1}\right) \rightarrow 0 .
$$

But the cokernel of the first arrow is precisely $H^{1}(X, T X)$, at least if we have $H^{1}\left(X, T \Sigma_{\mid X}\right)=0$ or, by Serre duality again, $H^{d_{X}-1}\left(X, \Omega_{\Sigma}^{d_{X}} \otimes L_{\mid X}^{-\iota}\right)=0$. This follows from (3).

Of course we can easily imagine variants if this lemma for complete intersections, or even more generally, for zero-loci of global sections of vector bundles. For the construction to work, we need a huge number of vanishing conditions on the ambient variety $\Sigma$, and it is not so clear a priori that we can find any example that way. We have been able to find some under the hypothesis that $\Sigma$ is a homogeneous space.

3.3. Linear sections of homogeneous spaces. Suppose that $\Sigma$ is a rational homogeneous variety, and that $\iota=\iota_{\Sigma}$ is equal to its index. In particular we require that the dimension of $\Sigma$ is $d_{\Sigma}=2 \iota_{\Sigma}+2$. There is a short list of suitable examples:

$\begin{array}{cccc}\Sigma & \text { dim } & \text { index } & \text { moduli } \\ \left(\mathbb{P}^{1}\right)^{6} & 6 & 2 & 45 \\ \left(\mathbb{P}^{1}\right)^{3} \times \mathbb{P}^{3} & 6 & 2 & 55 \\ \left(\mathbb{P}^{2}\right)^{4} & 8 & 3 & 48 \\ \left(\mathbb{P}^{4}\right)^{3} & 12 & 5 & 52 \\ G(2,5) \times G(2,5) & 12 & 5 & 51 \\ G(4,9) & 20 & 9 & 46 \\ G(3,11) & 24 & 11 & 45\end{array}$


Proposition 3.2. Let $\Sigma$ belong to the list above, and $X=\Sigma \cap H$ be a smooth hyperplane section of $\Sigma$ in its minimal complete embedding. Then $X$ is of Calabi-Yau type.

Proof. We check case by case that the vanishing conditions of Lemma 3.1 do hold. For Grassmannians this requires the diagrammatic methods first developed in $\mathrm{Sn}$.

3.4. Quadratic sections of homogeneous spaces. Now suppose that $\Sigma$ is again a rational homogeneous variety of even index $\iota_{\Sigma}$, and let $\iota=\iota_{\Sigma} / 2$. The relation $d_{\Sigma}=2 \iota+2$ becomes $\iota_{\Sigma}=d_{\Sigma}-2$, which is the definition of the Mukai varieties. It has been proved that apart from complete intersections, Mukai varieties are linear sections of homogeneous varieties (note that a linear section of a Mukai variety, if Fano, is again a Mukai variety), or quadric sections of the cone over $G(2,5)$. The homogeneous Mukai varieties are the following:

$\begin{array}{cccc}\Sigma & \text { dim } & \text { index } & \text { moduli } \\ \left(\mathbb{P}^{1}\right)^{4} & 4 & 2 & 68 \\ G_{2}^{a d} & 5 & 3 & 62 \\ \mathbb{P}^{3} \times \mathbb{P}^{3} & 6 & 4 & 69 \\ L G(3,6) & 6 & 4 & 62 \\ I G(2,6) & 7 & 5 & 68 \\ G(2,6) & 8 & 6 & 69 \\ \mathbb{S}_{10} & 10 & 8 & 80\end{array}$

In this table we have denoted by $I G(k, 2 n)$ the symplectic Grassmannian, parametrizing isotropic $k$-dimensional subspaces of a $2 n$-dimensional vector space endowed with a non-degenerate skew-symmetric form. When $k=n$ we rather use the notation $L G(n, 2 n)$ and call this variety the Lagrangian Grassmannian. Similarly, $\mathbb{S}_{2 n}$ denotes the spinor variety, which parametrizes one of the two families of isotropic $n$-dimensional subspaces of a $2 n$-dimensional vector space endowed with a non-degenerate quadratic form. The terminology comes from the fact that the minimal equivariant embedding of this variety is inside a projectivized half-spin representation - a quadratic Veronese is required in order to recover the Plücker embedding. Finally, the adjoint variety $G_{2}^{a d}$ denotes the closed $G_{2}$-orbit inside the projectivized adjoint representation.

Proposition 3.3. Let $X=\Sigma \cap Q$ be a smooth quadric section of a homogeneous Mukai variety of even dimension. Then $X$ is of Calabi-Yau type.

Proof. Again we need to check, case by case, the conditions of Lemma 3.1. Let us treat in some detail the case of $\mathbb{S}_{10}$, which is the most complicated one. Here $X$ is nine dimensional, and the claim is that its middle dimensional Hodge numbers are

$$
h^{9,0}=h^{8,1}=h^{7,2}=0, \quad h^{6,3}=1, \quad h^{5,4}=80 .
$$


Let $E$ denote the tautological rank five vector bundle on $\Sigma=\mathbb{S}_{10}$. The cotangent bundle of $\Sigma$ is $\Omega_{\Sigma}^{1} \simeq \wedge^{2} E$. In higher degree, the bundle $\Omega_{\Sigma}^{p}$ of $p$-forms can have several components, which are Schur powers of $E$ obtained as follows. Let $a=\left(a_{0}>a_{1}>\cdots>a_{m}\right)$ be a decreasing sequence of integers, whose sum is equal to $p$. Define the sequence

$$
\lambda(a)=\left(a_{0}, a_{1}+1, \ldots, a_{m}+m, m^{a_{m-1}-a_{m}-1}, \ldots, 1^{a_{0}-a_{1}-1}\right) .
$$

Then by the results of [Ko], $\Omega_{\Sigma}^{p}=\wedge^{p}\left(\wedge^{2} E\right)$ is the direct sum of the Schur powers $S_{\lambda(a)} E$. Note that since $E$ has rank five, the number of non zero components of $\lambda(a)$ must not exceed five, which means that $a_{0}-a_{m} \leq 4$.

Bott's theorem allows to compute the cohomology groups of a Schur power $S_{\lambda} E$ of the tautological bundle, for $\lambda=\left(\lambda_{1}, \ldots, \lambda_{5}\right)$ a non increasing sequence of non negative numbers. The rule is the following. Suppose that there exists a pair $(i, j)$ of distinct indices such that $\lambda_{i}+\lambda_{j}=i+j-2$. Then $S_{\lambda} E$ is acyclic. Otherwise, let $q$ be the number of pairs such that $\lambda_{i}+\lambda_{j}>i+j-2$. Then $S_{\lambda} E$ has a unique non-zero cohomology group, that of degree $q$.

Applying these rules, it is easy to check the following statement.

Lemma 3.4. The following bundles of twisted forms on $\Sigma$ are acyclic:

(1) $\Omega_{\Sigma}^{1}(-k)$ for $k=1,2,3$.

(2) $\Omega_{\Sigma}^{2}(-k)$ for $k=1,2,3$.

(3) $\Omega_{\Sigma}^{3}(-k)$ for $k=1,2$.

(4) $\Omega_{\Sigma}^{4}(-k)$ for $k=1,2$.

Moreover, for $k \geq 4, \Omega_{\Sigma}^{1}(-k)$ and $\Omega_{\Sigma}^{2}(-k)$ have non-zero cohomology groups only in degree $\operatorname{dim} \Sigma$. And the same is true for $\Omega_{\Sigma}^{3}(-k)$ and $\Omega_{\Sigma}^{4}(-k)$ when $k \geq 3$.

This is precisely what we need in order to apply Lemma 3.1 .

3.5. A quasi-homogeneous Mukai variety. Consider a hyperplane section $\Theta$ of the Lagrangian Grassmannian $L G(3,6)$. This is a five dimensional variety of index three.

Proposition 3.5. The variety $\Theta$ has the following properties:

(1) it is a quasi-homogeneous $P S L_{3}$-variety;

(2) its non-zero Hodge numbers are $h^{p, p}(\Theta)=1,1 \leq p \leq 5$;

(3) it is a minimal compactification of $\mathbb{C}^{5}$;

(4) it is rigid.

Proof. Consider the Lagrangian Grassmannian $L G(3,6)$ in its minimal homogeneous embedding $\mathbb{P} V=\mathbb{P}^{13}$. The orbit structure of $S p_{6}$ in $\mathbb{P} V$ is well-known, in particular it is prehomogeneous: there exists an open orbit, and the generic stabilizer is $P S L_{3}$ (up to a finite group). Since $V$ is selfdual, we conclude that the general hyperplane section $\Theta$ is rigid and admits a $P S L_{3}$-action. 
An explicit computation shows that $P S L_{3}$ has four orbits in $\Theta$. This can be seen as follows. The Lagrangian Grassmannian $L G(3,6)$ is known to be one of the few homogeneous varieties with one apparent double point. This means that a general point of $\mathbb{P} V$ belongs to a unique secant $\overline{x y}$, where $x$ and $y$ represent two generic isotropic planes. The orbits in $\Theta$ are then determined by the relative position with respect to $x$ and $y$, that is, by the dimensions of these intersections. These can be $(0,0)$ (the generic case, giving the open orbit), $(1,1)$ (a hyperplane section), $(2,1)$ or $(1,2)$ (two Veronese surfaces).

The computation of the Hodge numbers is straightforward. Finally, it is easy to check that a maximal torus in $P S L_{3}$ has a finite number of fixed points in $\Theta$. The Byalinicki-Birula decomposition $[\mathrm{BB}$ then ensures that $\Theta$ is a compactification of $\mathbb{C}^{5}$, and it is clearly minimal.

3.6. Double coverings. Consider a double covering $Y \rightarrow \Sigma$, branched over a smooth hypersurface $X$ of $\Sigma$, of degree $2 d$. The Hodge numbers of $Y$ can be computed in terms of the pair $(\Sigma, X)$ as follows [Cy]:

$$
h^{p, q}(Y)=h^{p, q}(\Sigma)+h^{q}\left(\Omega_{\Sigma}^{p}(\log X)(-d)\right) .
$$

Recall that $\Omega_{\Sigma}^{p}(\log X)$ denotes the vector bundle of logarithmic $p$-forms on $\Sigma$ with simple poles at most on $X$. There is an exact sequence

$$
0 \rightarrow \Omega_{\Sigma}^{p} \rightarrow \Omega_{\Sigma}^{p}(\log X) \rightarrow \Omega_{X}^{p-1} \rightarrow 0
$$

defined by taking residues on $X$.

Suppose that $\Sigma$ is homogeneous of odd dimension $n=2 m+1$. In particular the Hodge structure of $\Sigma$ is pure and we get $h^{p, q}(\Sigma)=0$ for $p+q=n$. Suppose moreover that $H^{q}\left(\Sigma, \Omega_{\Sigma}^{p}(-d)\right)=H^{q+1}\left(\Sigma, \Omega_{\Sigma}^{p}(-d)\right)=0$. Then $h^{p, q}(Y)=h^{q}\left(X, \Omega_{X}^{p-1}(-d)\right)$, which can be computed by considering a long exact sequence like in the proof of Lemma 3.1. Under favorable circumstances, we expect to derive that

$$
h^{m+2, m-1}(Y)=h^{m+2}\left(X, \Omega_{X}^{m-2}(-d)\right)=h^{2 m}\left(X, \mathcal{O}_{X}(-d-(m-2) 2 d)\right)=\mathbb{C}
$$

if moreover $\omega_{X}=\mathcal{O}_{X}(-(2 m-3) d)$, that is, $\omega_{\Sigma}=\mathcal{O}_{\Sigma}(-(2 m-1) d)$. If we let $d=1$, this means that $\Sigma$ is a Mukai variety. If we let $\Sigma$ be homogeneous, or the quasi-homogeneous Mukai variety of the previous section, we check that the expected vanishing theorems do hold and we arrive at the following conclusion.

Proposition 3.6. Let $Y \rightarrow \Sigma$ be a double covering of a homogeneous or quasi-homogeneous Mukai variety of odd dimension, branched over a smooth quadric section. Then $Y$ is a Fano manifold of Calabi-Yau type.

3.7. The Cayley trick. Let $X \subset \mathbb{P}^{N}$ be a complete intersection of multidegree $\left(d_{1}, \ldots, d_{m}\right)$, where none of these degrees equals one. Let $L_{i}=\mathcal{O}_{\mathbb{P}^{N}}\left(d_{i}\right)$ and $P=\mathbb{P}\left(E^{\vee}\right)$, where $E=L_{1} \oplus \cdots \oplus L_{m}$. The equations of $X$ can be considered as a section $\sigma$ in $H^{0}\left(\mathbb{P}^{N}, E\right) \simeq H^{0}\left(P, \mathcal{O}_{E}(1)\right)$. Hence an associated divisor $\mathcal{X} \subset P$, which is smooth when $X$ is smooth. By [Na, Lemma 2.7], 
the cohomology of $\mathcal{X}$ is essentially the same as that of $X$, with a shift by $2 m-2=\operatorname{dim} \mathcal{X}-\operatorname{dim} X$ in the degrees.

Moreover the deformations of $X$ and $\mathcal{X}$ are both unobstructed, and

$$
H^{1}(X, T X) \simeq H^{1}(\mathcal{X}, T \mathcal{X}) .
$$

Indeed, the left hand side of (11) is given by the exact sequence

$$
H^{0}\left(X, T \mathbb{P}_{\mid X}^{N}\right) \rightarrow H^{0}\left(X, E_{\mid X}\right) \rightarrow H^{1}(X, T X) \rightarrow 0 .
$$

It is easy to check that $H^{0}\left(X, T \mathbb{P}_{\mid X}^{N}\right) \simeq H^{0}\left(\mathbb{P}^{N}, T \mathbb{P}^{N}\right)=\mathfrak{s l}_{N}$. Moreover, the Koszul complex of the section $\sigma$ of $E$ defining $X$ shows that $H^{0}\left(X, E_{\mid X}\right)$ is just the cokernel of the natural map

$$
H^{0}\left(\mathbb{P}^{N}, \operatorname{End}(E)\right) \rightarrow H^{0}\left(\mathbb{P}^{N}, E\right)
$$

defined by applying an endomorphism of $E$ to the section $\sigma$.

Similarly, the right hand side of (1) is given by an exact sequence

$$
H^{0}\left(\mathcal{X}, T P_{\mid \mathcal{X}}\right) \rightarrow H^{0}\left(\mathcal{X}, \mathcal{O}_{\mathcal{X}}(1)\right) \rightarrow H^{1}(\mathcal{X}, T \mathcal{X}) \rightarrow 0 .
$$

We have $H^{0}\left(\mathcal{X}, \mathcal{O}_{\mathcal{X}}(1)\right) \simeq H^{0}\left(P, \mathcal{O}_{E}(1)\right) / \mathbb{C} \sigma=H^{0}\left(\mathbb{P}^{N}, E\right) / \mathbb{C} \sigma$. Moreover, $H^{0}\left(\mathcal{X}, T P_{\mid \mathcal{X}}\right)=H^{0}(P, T P)$ can be computed through the differential of the projection $\pi$ from $P$ to $X$ and the description of its kernel, the vertical tangent space $T^{v} P$, by the relative Euler sequence

$$
0 \rightarrow \mathcal{O}_{P} \rightarrow \pi^{*} E^{\vee} \otimes \mathcal{O}_{P}(1) \rightarrow T^{v} P \rightarrow 0 .
$$

We get that $H^{0}(P, T P)$ is the direct sum of $H^{0}\left(P, T^{v} P\right)$ and $\mathfrak{s l}_{N}$, while $H^{0}\left(P, T^{v} P\right) \simeq H^{0}\left(\mathbb{P}^{N}, E_{n} d_{0}(E)\right)$. Since the maps $H^{0}\left(\mathbb{P}^{N}, E n d(E)\right) \rightarrow$ $H^{0}\left(\mathbb{P}^{N}, E\right)$ and $H^{0}\left(\mathbb{P}^{N}, E n d_{0}(E)\right) \rightarrow H^{0}\left(\mathbb{P}^{N}, E\right) / \mathbb{C} \sigma$ have the same cokernels, the identification (11) follows. We conclude:

Proposition 3.7. If $X \subset \mathbb{P}^{N}$ is a Calabi-Yau manifold, or a complete intersection of Calabi-Yau type, then $\mathcal{X} \subset P$ is also of Calabi-Yau type.

\section{A special Series of manifolds of CAlabi-Yau type}

4.1. A special non-vanishing. Consider the following series of homogeneous varieties $\Sigma$ :

$\begin{array}{ccccc}\Sigma & \text { dim } & \text { index } & \text { coindex } & \text { deg } \\ \mathbb{O P}^{2} & 16 & 12 & 4 & 3 \\ \mathbb{S}_{12} & 15 & 10 & 5 & 4 \\ G(2,10) & 16 & 10 & 6 & 5 \\ \mathbb{S}_{14} & 21 & 12 & 9 & 8\end{array}$

Here we denoted by $\mathbb{O P}^{2}$ the so-called Cayley plane, which is the unique Hermitian symmetric space of type $E_{6}$. Recall that the minimal representation of $E_{6}$ has dimension 27. They Cayley plane can be obtained as the minimal $E_{6}$-orbit in the projectivization of this representation (or its dual). 
For more on the extraordinary properties of this variety, see [IM1] and the references therein.

Recall that the index $r$ is defined by the identity $K_{\Sigma}=-r H$, where $H$ is the ample generator of the Picard group of $\Sigma$. The coindex is the difference between the dimension and the index.

The last column gives the degree of the dual variety of $\Sigma$. Note that this degree is always one less than the coindex, a coincidence that will play a major role below.

Lemma 4.1. Let $\Sigma$ be as above, with index $r$ and coindex $c$. Then

$$
H^{r+2}\left(\Sigma, \Omega_{\Sigma}^{c-2}(c-r-1)\right)=\mathbb{C} .
$$

Proof. This is a straightforward application of the Borel-Weil-Bott theorem. The details are provided in the Appendix.

4.2. Relation with projective duality. The explanation for the nonvanishing of Lemma 4.1 is the following. We start by recalling the usual setting of projective duality, which we summarize in the diagram

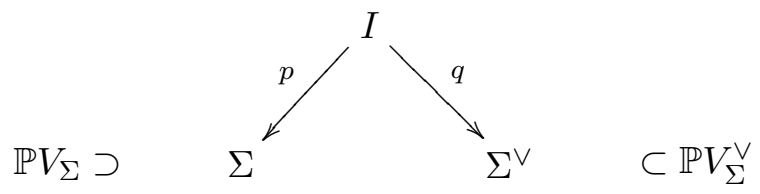

where we denoted by $I \subset \mathbb{P} V_{\Sigma} \times \mathbb{P} V_{\Sigma}^{\vee}$ the incidence variety, defined as the set of pairs $(x, h)$ with $x \in \Sigma$ and $h$ a hyperplane containing the affine tangent space to $\Sigma$ at $h$. The variety $\Sigma^{\vee}$ parametrizing tangent hyperplanes to $\Sigma$ is its projective dual variety. For all the cases we are interested in, the dual variety is non-degenerate, which means that it is a hypersurface whose degree has been computed in the framework of prehomogeneous vector spaces.

Indeed, the variety $\Sigma$ is the closed orbit of the simple group $\operatorname{Aut}(\Sigma)$ in a (projectivized) prehomogeneous space $\mathbb{P}\left(V_{\Sigma}\right)$ whose orbit structure is known explicitly. Part of the structure is common to all cases: there is of course an open orbit, its complement is an irreducible hypersurface, and this hypersurface contains an orbit closure $W_{\Sigma}$ whose complement is again a single orbit. This can be observed case by case.

If $\Sigma$ is the Cayley plane, there are only three orbits in $\mathbb{P}\left(V_{\Sigma}\right)$, the variety $\Sigma$ itself, its complement in its secant variety, which is a cubic hypersurface, and the complement of this hypersurface. In particular $W_{\Sigma}=\Sigma$ in that case. An equation of the $E_{6}$-invariant cubic was first written down by Elie Cartan (in terms of the geometry of the 27 lines on a smooth cubic surface) and we therefore call it the Cartan cubic.

If $\Sigma=\mathbb{S}_{12}$, there are four orbits [Ig], whose closures are the whole projective space, a quartic hypersurface, $\Sigma$ itself, and an intermediate codimension seven variety which is our $W_{\Sigma}$.

If $\Sigma=G(2,10)$, embedded in the projectivization of the space of skewsymmetric forms, there are five orbits determined by the ranks of the forms; 
the invariant hypersurface is defined by the Pfaffian; $W_{\Sigma}$ is the space of forms of rank six at most, its codimension is six.

Finally, if $\Sigma=\mathbb{S}_{14}$, there are exactly nine orbits which were first described by Popov $[\mathrm{Po}]$. His results show that $W_{\Sigma}$ has codimension five.

In each case we expect $W_{\Sigma}$ to coincide with the singular locus of the invariant hypersurface. At least we know that it contains this singular locus, just because of the orbit structure.

The orbit structure is the same in the dual projective space $\mathbb{P}\left(V_{\Sigma}^{\vee}\right)$, in particular the invariant hypersurface is the projective dual variety of $\Sigma$. We summarize the relevant data in the following statement. We have no explanation, even conjectural, of these curious numerical coincidences.

Lemma 4.2. The projective dual $\Sigma^{\vee}$ to $\Sigma$ is a hypersurface of degree $c-1$, whose singular locus has codimension at least $r-c+2$.

The following table gives $V_{\Sigma}$ for each case and the dual hypersurface $\Sigma^{\vee}$.

$\begin{array}{lll}\Sigma & V_{\Sigma} & \Sigma^{\vee} \\ \mathbb{O P}^{2} & H_{3}(\mathbb{O}) & \text { Cartan cubic } \\ \mathbb{S}_{12} & \Delta_{+} & \text {Igusa quartic } \\ G(2,10) & \wedge^{2} \mathbb{C}^{10} & \text { Pfaffian quintic } \\ \mathbb{S}_{14} & \Delta_{+} & \text {Popov octic }\end{array}$

Since the quotient of $V_{\Sigma}$ by the affine tangent bundle is the twisted normal bundle $N(-1)$, we have a natural identification $I \simeq \mathbb{P}\left(N(-1)^{\vee}\right)$. Moreover this identification is such that the relative tautological bundle $\mathcal{O}_{N(-1)}(1)$ coincides with $q^{*} \mathcal{O}(1)$.

Now, the duality theorem (see e.g. [GKZ) asserts that $\left(\Sigma^{\vee}\right)^{\vee}=\Sigma$, and moreover, that the diagram above is symmetric in the sense that $I$ can be defined as parametrizing the tangent hyperplanes to the hypersurface $\Sigma^{\vee}$ (at least at smooth points, and then one needs to take the Zariski closure). If we denote by $F_{\Sigma} \in S y m^{c-1} V_{\Sigma}$ an equation of $\Sigma^{\vee}$, this means that $I$ can be obtained as the closure of the set of points $\left(\left[d F_{\Sigma}(\xi)\right],[\xi]\right)$, where $\xi$ belongs to the cone over $\Sigma_{\text {reg }}^{\vee}$. This means in particular that we have a non-zero section

$$
\delta F_{\Sigma} \in H^{0}\left(I, \operatorname{Hom}\left(q^{*} \mathcal{O}(-c+2), p^{*} \mathcal{O}(-1)\right)\right),
$$

vanishing precisely over the singular locus of $\Sigma^{\vee}$. Pushing this section forward to $\Sigma$, we get a section

$$
\left.\bar{\delta} F_{\Sigma} \in H^{0}\left(\Sigma, \operatorname{Sym}^{c-2}(N(-1))(-1)\right)=H^{0}\left(\Sigma, \operatorname{Sym}^{c-2} N(-c+1)\right)\right) .
$$

Now we can use the normal exact sequence

$$
0 \rightarrow T \Sigma \rightarrow T \mathbb{P}\left(V_{\Sigma}\right) \otimes \mathcal{O}_{\Sigma} \rightarrow N \rightarrow 0 .
$$

Taking a suitable wedge power and twist, we get a long exact sequence

$$
\left.0 \rightarrow \wedge^{c-2} T \Sigma(-c+1) \rightarrow \cdots \rightarrow \text { Sym }^{c-2} N(-c+1)\right) \rightarrow 0 .
$$


A careful check would then allow to conclude that this long exact sequence induces an isomorphism

$$
\left.H^{0}\left(\Sigma, \operatorname{Sym}^{c-2} N(-c+1)\right)\right) \simeq H^{c-2}\left(\Sigma, \wedge^{c-2} T \Sigma(-c+1)\right),
$$

and the latter is Serre dual to $H^{r+2}\left(\Sigma, \Omega_{\Sigma}^{c-2}(c-r-1)\right)$. In particular, we see that that our non-zero section $\bar{\delta} F_{\Sigma}$ induces a non-zero linear form on this one dimensional cohomology group.

4.3. Linear sections. Let $X$ be a general linear section of $\Sigma$, of codimension $s=r-c+1$. Then $X$ has dimension $n=2 c-1$ and index $c-1$. The following statement is a special case of a result of Borcea [B]]:

Lemma 4.3. Any small deformation of $X$ is a linear section of $\Sigma$.

In particular, the number of moduli for $X$ is the dimension $m$ of the quotient of the Grassmannian $G\left(s, V_{\Sigma}\right)$ by the simple group Aut $(\Sigma)$, of dimension $\delta$. The relevant data appear in the table below.

$\begin{array}{cccccc}\Sigma & s & N & \text { Aut }(\Sigma) & \delta & m \\ \mathbb{O P}^{2} & 9 & 26 & E_{6} & 78 & 84 \\ \mathbb{S}_{12} & 6 & 31 & \text { Spin }_{12} & 66 & 90 \\ G(2,10) & 5 & 44 & \text { PSL }_{10} & 99 & 101 \\ \mathbb{S}_{14} & 4 & 63 & \text { Spin }_{14} & 91 & 149\end{array}$

Theorem 4.4. A generic linear section $X$ of $\Sigma$ has primitive cohomology only in middle degree. Its non-zero Hodge numbers $h^{p, n-p}$, for $p \geq c$, are the following:

$$
h^{c+1, c-2}=1, \quad h^{c, c-1}=m .
$$

Proof. Let us denote by $L \subset V_{\Sigma}^{\vee}$ the space of linear forms that defines $X$. Its dimension is $s=r-c+1$. We use the conormal sequence

$$
0 \rightarrow \mathcal{O}_{X}(-1) \otimes L \rightarrow \Omega_{\Sigma \mid X}^{1} \rightarrow \Omega_{X}^{1} \rightarrow 0
$$

and its wedge powers, for positive integers $p$,

$$
0 \rightarrow \mathcal{O}_{X}(-p) \otimes \operatorname{Sym}^{p} L \rightarrow \cdots \rightarrow \Omega_{\Sigma \mid X}^{p-1}(-1) \otimes L \rightarrow \Omega_{\Sigma \mid X}^{p} \rightarrow \Omega_{X}^{p} \rightarrow 0 .
$$

We claim that for $p \leq c-1$, this induces an isomorphism

$$
H^{q}\left(X, \Omega_{\Sigma \mid X}^{p}\right) \simeq H^{q}\left(X, \Omega_{X}^{p}\right) .
$$

To check this, we will prove that the other vector bundles involved in the long exact sequence above, are all acyclic. These bundles are the bundles of twisted forms $\Omega_{\Sigma \mid X}^{p-k}(-k)$, for $1 \leq k \leq p$. For example, for $k=p$ we observe that

$$
H^{q}\left(X, \mathcal{O}_{X}(-p)\right)=0 \quad \forall q \leq n-1
$$

by Kodaira's vanishing theorem, while by Serre duality

$$
H^{q}\left(X, \mathcal{O}_{X}(-p)\right)=H^{n-q}\left(X, \mathcal{O}_{X}(p-c+1)\right)^{\vee}=0 \quad \forall q \geq 1
$$


when $p \leq c-2$. For the remaining terms, we use the Koszul resolution of the structure sheaf of $X$,

$$
0 \rightarrow \mathcal{O}_{\Sigma}(c-r-1) \otimes \wedge^{r-c+1} L \rightarrow \cdots \rightarrow \mathcal{O}_{\Sigma}(-1) \otimes L \rightarrow \mathcal{O}_{\Sigma} \rightarrow \mathcal{O}_{X} \rightarrow 0 .
$$

Twisting this long exact sequence by $\Omega_{\Sigma}^{p-k}(-k)$, we see that in order to prove the acyclicity of $\Omega_{\Sigma \mid X}^{p-k}(-k)$, it is enough to check the acyclicity of $\Omega_{\Sigma}^{p-k}(-k-\ell)$ for $0 \leq \ell \leq r-c+1$. But this follows from Lemma 5.1.

By the same Lemma, $\Omega_{\Sigma}^{p}(-\ell)$ is acyclic for $1 \leq \ell \leq r-c+1$ and $p \leq c-3$. This implies that

$$
H^{q}\left(\Sigma, \Omega_{\Sigma}^{p}\right) \simeq H^{q}\left(X, \Omega_{\Sigma \mid X}^{p}\right) \simeq H^{q}\left(X, \Omega_{X}^{p}\right)
$$

for all $q$ and for all $p \leq c-2$.

For $p=c-2$ there is a unique non acyclic bundle involved, $\Omega_{\Sigma}^{c-2}(c-r-1)$ : by Lemma 4.1, this bundle has a one-dimensional cohomology group in degree $r+2$, but the other cohomology groups vanish. Then the Koszul resolution of $\mathcal{O}_{X}$ implies that

$$
H^{q}\left(X, \Omega_{\Sigma \mid X}^{c-2}\right)_{0}=H^{q+r-c+1}\left(\Sigma, \Omega_{\Sigma}^{c-2}(c-r-1)\right)=\delta_{q, c+1} \mathbb{C} .
$$

Finally, for $p=c-1$ we find two non-zero cohomology groups for the bundles involved in the complex

$$
0 \rightarrow \mathcal{O}_{X}(1-c) \otimes S y m^{c-1} L \rightarrow \cdots \rightarrow \Omega_{\Sigma \mid X}^{c-2}(-1) \otimes L \rightarrow \Omega_{\Sigma \mid X}^{c-1} \rightarrow \Omega_{X}^{c-1} \rightarrow 0 .
$$

Indeed, by Lemma $5.1 \Omega_{\Sigma}^{c-2}(c-r)$ is acyclic. Then, by the same argument as above, the cohomology of the bundle $\Omega_{\Sigma \mid X}^{c-2}(-1)$ is

$$
H^{q}\left(X, \Omega_{\Sigma \mid X}^{c-2}(-1)\right)=H^{q+r-c}\left(\Sigma, \Omega_{\Sigma}^{c-2}(c-r-1) \otimes \wedge^{r-c} L\right)=\delta_{q, c+2} L^{\vee} .
$$

On the other hand, the line bundle $\mathcal{O}_{X}(1-c)$ is the canonical bundle of $X$, and therefore it has non trivial cohomology in degree $n=2 c-1$, and only in that degree. Note that $\Omega_{\Sigma \mid X}^{c-1}$ also has non-zero cohomology, but only in degree $c-1$, fo which we get the same cohomology group as for $\Omega_{\Sigma}^{c-1}$. We deduce a long exact sequence

$$
\begin{aligned}
\cdots \rightarrow H^{q}\left(X, \Omega_{X}^{c-1}\right)_{0} & \rightarrow H^{q+c-1}\left(X, \omega_{X}\right) \otimes S^{2-1} m^{c-1} L \rightarrow \\
& \rightarrow H^{q+2}\left(X, \Omega_{\Sigma \mid X}^{c-2}(-1)\right) \otimes L \rightarrow H^{q+1}\left(X, \Omega_{X}^{c-1}\right)_{0} \rightarrow \cdots
\end{aligned}
$$

But note that this long exact sequence has very few non-zero terms, appearing precisely for $q=c$. Note also that by Hodge symmetry, we already know that $H^{c+1}\left(X, \Omega_{X}^{c-1}\right)=H^{c}\left(X, \Omega_{X}^{c-2}\right)=0$. What remains is the short exact sequence

$$
0 \rightarrow H^{c}\left(X, \Omega_{X}^{c-1}\right) \rightarrow \operatorname{Sym}^{c-1} L \rightarrow L^{\vee} \otimes L \rightarrow 0,
$$

from which we can easily compute $h^{c, c-1}$. And we conclude, as claimed, that $h^{c, c-1}=m$.

Remark. In general, if $\Sigma$ has a non degenerate dual of degree $d \leq n$, we could expect $H^{n+1-d}\left(\Sigma, \Omega_{\Sigma}^{d-1}(d-r)\right)$ to be non-zero, and even one-dimensional. 
Under favorable circumstances, this should imply that if $X$ is a general linear section of codimension $r-d$, hence dimension $c+d$, then $h^{c+1}\left(\Omega_{X}^{d-1}\right)=1$.

4.4. Deformations. Observe that the conclusion of Theorem 4.4 has been obtained by a case by case computation, as a coincidence between two numbers that were computed from quite different, and at first sight, unrelated data.

On one hand, the number $m$ of moduli has been computed as the difference between the dimension of a suitable Grassmannian and that of the automorphism group of $\Sigma$. Indeed, $H^{1}(X, T X)$ is given by the exact sequence

$$
0 \rightarrow H^{0}\left(X, T \Sigma_{\mid X}\right) \rightarrow H^{0}\left(X, L^{\vee}(1)\right) \rightarrow H^{1}(X, T X) \rightarrow 0 .
$$

It is straightforward to check that

$$
H^{0}\left(X, T \Sigma_{\mid X}\right)=H^{0}(\Sigma, T \Sigma)=\operatorname{aut}(\Sigma),
$$

the Lie algebra of $\operatorname{Aut}(\Sigma)$, while $H^{0}\left(X, L^{\vee}(1)\right)=L^{\vee} \otimes V_{\Sigma}^{\vee} / L$ is the tangent space to the Grassmannian of subspaces of $V_{\Sigma}$, at its point defined by $L$. This tangent space is also the quotient of $g l\left(V_{\Sigma}\right)$ by the stabilizer of $L$, hence the identification

$$
H^{1}(X, T X) \simeq g l\left(V_{\Sigma}\right) /(\operatorname{aut}(\Sigma)+\operatorname{stab}(L)) .
$$

On the other hand, the exact sequence (2) shows that

$$
H^{c}\left(X, \Omega_{X}^{c-1}\right)^{\vee} \simeq S y m^{c-1} L^{\vee} / g l(L) .
$$

As we have seen, the connection between these two descriptions is provided by the equation $F_{\Sigma}$, of degree $c-1$, of the dual hypersurface $\Sigma^{\vee}$. We have a natural map from $g l\left(V_{\Sigma}\right)$ to $S y m^{c-1} V_{\Sigma}$, sending $u$ to $F_{\Sigma} \circ u$, then to Sym ${ }^{c-1} L^{\vee}$ by restriction to $L$. Since aut $(\Sigma)$ kills $F_{\Sigma}$, this induces a map

$$
g l\left(V_{\Sigma}\right) /(\operatorname{aut}(\Sigma)+\operatorname{stab}(L)) \rightarrow \operatorname{Sym}^{c-1} L^{\vee} / g l(L),
$$

which is an isomorphism if and only if $X$ is of Calabi-Yau type.

This has a modular interpretation: we can associate to $X$ the hypersurface $X^{\vee}=\mathbb{P} L \cap \Sigma^{\vee}$ (which is of course not the projective dual variety of $X$ ). By Lemma 4.2, this hypersurface will be smooth of degree $c-1$ for a generic $L$. Moreover, if $X^{\vee}$ has discrete automorphism group, the quotient $S y m^{c-1} L^{\vee} / g l(L)$ can be interpreted as $H^{1}\left(X^{\vee}, T X^{\vee}\right)$, Otherwise said, the correspondence between $X$ and $X^{\vee}$ defines a map from the moduli space of linear sections of $\Sigma$ to the moduli space of degree $c-1$ hypersurfaces in $\mathbb{P}^{r-c}$, and the morphism (3) is nothing else than the differential of that map. We deduce:

Proposition 4.5. The variety $X$ is a Fano manifold of Calabi-Yau type if and only if its small deformations induce a versal family of degree $c-1$ hypersurfaces in $\mathbb{P}^{r-c}$. 
Note that if this is true, we can conclude that a generic hypersurface of degree $c-1$ in $\mathbb{P}^{r-c}$ can be defined, up to isomorphism, as a linear section of $\Sigma^{\vee}$ in a finite (non-zero) number of different ways.

For the quintic threefold, it has already been noticed by Beauville that there exists finitely many Pfaffian representations (see Schreyer's Appendix to [B] ). Beauville asked how many such representations do exist : in principle the answer is given by some Donaldson-Thomas invariant. We can ask the same question in our three other cases. The case of cubic sevenfolds is treated in IM2] where we prove that the very same phenomena hold. That's also what we expect for the two remaining cases, which will deserve further investigations.

It is also quite remarkable that we can associate to $X$ either a CalabiYau threefold, or another Fano manifold of Calabi-Yau type, by considering either the dual $X^{\vee}$, or a double covering of $\mathbb{P}^{r-c}$, branched over $X^{\vee}$. We get the following varieties $X^{*}$ :

$$
\begin{array}{ll}
X & X^{*} \\
\mathbb{O P}^{2} \cap \mathbb{P}^{17} & \text { cubic sevenfold } \\
\mathbb{S}_{12} \cap \mathbb{P}^{25} & \text { double quartic fivefold } \\
G(2,10) \cap \mathbb{P}^{40} & \text { quintic threefold } \\
\mathbb{S}_{14} \cap \mathbb{P}^{59} & \text { double octic threefold }
\end{array}
$$

The last two varieties are famous examples of Calabi-Yau threefolds. The first two were among our very first examples of Fano manifolds of Calabi-Yau type. They are not Calabi-Yau's stricto sensu but, considered as (compactified) Landau-Ginzburg models, we have seen that they appeared in the litterature as mirrors to certain rigid Calabi-Yau threefolds [CDP, Sch].

Note that if the resulting map $\mathcal{K}_{X} \rightarrow \mathcal{K}_{X^{*}}$ from the base of the Kuranishi family of $X$, to that of $X^{*}$, is etale, then we have the same property for the gauged families and we can pull-back the integrable system in intermediate Jacobians of $X^{*}$ and its deformations (see Theorem 2.2). Although we did not check this, it is quite likely that we should recover the integrable system in intermediate Jacobians of $X$ itself and its deformations. In particular the intermediate Jacobians of $X$ and $X^{*}$ should be isomorphic.

4.5. Homological projective duality. We expect that our series of examples of Fano manifolds of Calabi-Yau type should give rise to interesting instances of homological projective duality [Ku2]. More precisely, the derived categories of $X$ and $X^{*}$ should both contain a three-dimensional Calabi-Yau category, say $\mathcal{A}_{X} \subset \mathcal{D}^{b}(\operatorname{Coh}(X))$ and $\mathcal{A}_{X^{*}} \subset \mathcal{D}^{b}\left(\operatorname{Coh}\left(X^{*}\right)\right)$ and there should exist a natural equivalence between $\mathcal{A}_{X}$ and $\mathcal{A}_{X^{*}}$.

For $X^{*}$ this was observed by Kuznetsov:

Proposition 4.6. Let $Y$ be among a the following types of Fano manifolds of Calabi-Yau type:

(1) a cubic sevenfold, 
(2) a cubic hypersurface in a six-dimensional quadric,

(3) a double-cover of $\mathbb{P}^{5}$ branched over a smmoth quartic.

Then the derived category $\mathcal{D}^{b}(Y)$ contains a natural subcategory $\mathcal{A}_{Y}$ which is a three-dimensional Calabi-Yau category.

Proof. For any Fano hypersurface $Y \subset \mathbb{P}^{n+1}$ of degree $d$, and index $\iota_{Y}=$ $n+2-d$, the collection

$$
\left\langle\mathcal{O}_{Y}, \ldots, \mathcal{O}_{Y}\left(\iota_{Y}-1\right)\right\rangle
$$

is obviously exceptional. Kuznetsov proved in [Ku1], Corollary 4.3, that if $d$ divides $n+2$, its left orthogonal $\mathcal{A}_{Y}$ is a Calabi-Yau category of dimension $n-2 \iota_{Y} / d$. In particular, for $d=3$ and $n=7$, we can conclude that $\mathcal{A}_{Y}$ is a three-dimensional Calabi-Yau category.

A very similar statement holds for double covers. Suppose that $Y$ is a double-cover of $\mathbb{P}^{n}$ branched over a general hypersurface of degree $2 d$. Then $Y$ is Fano for $d \leq n$, and $\omega_{Y}^{-1}=f^{*} \mathcal{O}_{\mathbb{P}^{n}}(n+1-d)$, so that $\iota_{Y}=n+1-d$. Here, for the same reason as before, the collection

$$
\left\langle\mathcal{O}_{Y}, f^{*} \mathcal{O}_{\mathbb{P}^{n}}(1), \ldots, f^{*} \mathcal{O}_{\mathbb{P}^{n}}\left(\iota_{Y}-1\right)\right\rangle
$$

is exceptional. Kuznetsov proved that its orthogonal is, when $d$ divides $n+1$, a Calabi-Yau category of dimension $n-\iota_{Y} / d$. For $d=2$ and $n=5$, we can conclude that $\mathcal{A}_{Y}$ is a three-dimensional Calabi-Yau category.

Finally the case of hypersurfaces in quadrics in very similar. In dimension 6 , the derived category has a Lefschetz decomposition $\mathcal{D}^{b}\left(\mathbb{Q}^{6}\right)=\langle A, A(3)\rangle$, where

$$
A=\left\langle S, O_{\mathbb{Q}^{6}}, O_{\mathbb{Q}^{6}}(1), O_{\mathbb{Q}^{6}}(2)\right\rangle,
$$

and $S$ denotes the rank four spin bundle. If $i: Y \hookrightarrow \mathbb{Q}^{6}$ is a cubic hypersurface, the functor $i^{*}: D^{b}\left(\mathbb{Q}^{6}\right) \rightarrow D^{b}(Y)$ is fully faithful on $A$ and the orthogonal $\mathcal{A}_{Y}$ to $i^{*}(A)$ in $D^{b}(Y)$ is a three-dimensional Calabi-Yau category.

The appearance of these three dimensional non-commutative Calabi-Yau's in connection with our manifolds of Calabi-Yau type (but not three dimensional!) is a quite remarkable phenomenon, which would deserve to be better understood.

\section{Appendix: Proof of Lemma 4.1}

First observe that, $\Sigma$ being a Hermitian symmetric space, its cotangent bundle is an irreducible homogeneous bundle. In particular its cohomology groups, and that of its twists as well, can be computed using Bott's theorem. Moreover the same is true for any bundle of $p$-forms on $\Sigma$, since it must split into a direct sum of irreducible homogeneous bundles.

Recall that an irreducible homogeneous vector bundle on a homogeneous space $\Sigma=G / P$ is defined by an irreducible representation of $P$, which is in turn defined by its highest weight: some weight $\lambda$ of $G$ which is $P$-dominant. We denote the corresponding bundle by $\mathcal{E}_{\lambda}$. 
When $\Sigma$ is a Hermitian symmetric space with Picard number one, $P$ is a maximal parabolic subgroup defined by some simple root $\alpha$, and the cotangent bundle is simply

$$
\Omega_{\Sigma}^{1}=\mathcal{E}_{-\alpha}
$$

More generally, a combinatorial formula has been obtained by Kostant for the irreducible components of each $\Omega_{\Sigma}^{p}$ [Ko.

Bott's theorem for an irreducible homogeneous vector bundle $\mathcal{E}_{\lambda}$ can be stated as the following recipe. Add $\rho$, the half-sum of the positive roots of $G$, to $\lambda$. If $\left\langle\lambda+\rho, \alpha^{\vee}\right\rangle=0$ for some coroot $\alpha^{\vee}$, then $\mathcal{E}_{\lambda}$ is acyclic. Otherwise, there is a unique $w$ in the Weyl group $W$ of $G$ such that $w(\lambda+\rho)=\mu+\rho$ for some dominant weight $\mu$. Then $\mathcal{E}_{\lambda}$ has a unique non-zero cohomology group, in degree $\ell(w)$, and it is an irreducible $G$-module of lowest weight $-\mu$. In particular, it is one dimensional if and only if $\mu=0$.

First case : $\Sigma=G(2,10)$. Here $\Omega_{\Sigma}^{1}=Q^{*} \otimes E$, where $E$ denotes the tautological rank two bundle and $Q$ the quotient rank eight bundle. The claim is that

$$
H^{12}\left(\Sigma, \Omega_{\Sigma}^{4}(-5)\right)=\mathbb{C}
$$

The decomposition of $\Omega_{\Sigma}^{4}$ into irreducible components is

$$
\Omega_{\Sigma}^{4}=\wedge^{4} Q^{*} \otimes S^{4} E \oplus S_{211} Q^{*} \otimes S_{31} E \oplus S_{22} Q^{*} \otimes S_{22} E .
$$

We apply Bott's theorem to each factor. The weights of the three factors are

$$
\begin{aligned}
& (0,0,0,0,-1,-1,-1,-1,4,0), \\
& (0,0,0,0,0,-1,-1,-2,3,1), \\
& (0,0,0,0,0,0,-2,-2,2,2) .
\end{aligned}
$$

Twisting by $\mathcal{O}_{\Sigma}(-5)$ and adding $\rho$ we get

$$
\begin{aligned}
& (4,3,2,1,-1,-2,-3,-4,5,0), \\
& (4,3,2,1,0,-2,-3,-5,4,1), \\
& (4,3,2,1,0,-1,-3,-4,3,2) .
\end{aligned}
$$

The last two sequences have repeated entries, indicating that the corresponding bundles are acyclic. But the first one has pairwise distinct entries, forming, once ordered, a consecutive sequence. This indicates that the corresponding bundle has one non-zero cohomology group, of dimension one, appearing in degree equal to the number of inversions of the sequence, which is twelve. This proves the claim.

Second case : $\Sigma=\mathbb{S}_{12}$. Here $\Omega_{\Sigma}^{1}=\wedge^{2} E$, where $E$ denotes the tautological rank six bundle. The claim is that

$$
H^{12}\left(\Sigma, \Omega_{\Sigma}^{3}(-6)\right)=\mathbb{C}
$$

The decomposition of $\Omega_{\Sigma}^{3}$ into irreducible components is

$$
\Omega_{\Sigma}^{3}=S_{411100} E \oplus S_{222000} E .
$$

Twisting by $\mathcal{O}_{\Sigma}(-6)$ and adding $\rho=(5,4,3,2,1,0)$ we get the weights $(2,1,0,-3,-4,-5)$ and $(2,0,-1,-2,-3,-7)$. The last sequence has two 
opposite entries, indicating that the corresponding bundle is acyclic. But this is not the case of the first sequence, whose absolute values form a consecutive sequence starting from zero. This implies that the corresponding bundle has a unique non-zero cohomology group, which is one dimensional. It appears in degree equal to the number of pairs of entries of the sequence whose sum is negative; this is twelve and the claim follows.

Third case : $\Sigma=\mathbb{S}_{14}$. Here again $\Omega_{\Sigma}^{1}=\wedge^{2} E$, where $E$ denotes the tautological rank seven bundle. The claim is that

$$
H^{14}\left(\Sigma, \Omega_{\Sigma}^{7}(-4)\right)=\mathbb{C} .
$$

The decomposition of $\Omega_{\Sigma}^{7}$ into irreducible components is

$$
\Omega_{\Sigma}^{7}=S_{6221111} E \oplus S_{5322110} E \oplus S_{4333100} E \oplus S_{4422200} E .
$$

Twisting by $\mathcal{O}_{\Sigma}(-7)$ and adding $\rho=(6,5,4,3,2,1,0)$ we get the weights

$$
\begin{aligned}
& (3,2,1,0,-2,-3,-8), \\
& (4,3,1,-2,-3,-4,-6), \\
& (4,2,1,-1,-2,-4,-7)), \\
& (4,3,0,-1,-2,-5,-6) .
\end{aligned}
$$

The first three have pairs of opposite entries, which implies that the corresponding bundles are acyclic. This is not the case of the last sequence, whose absolute values form a consecutive sequence starting from zero. As in the previous case, this implies the claim.

Fourth case : $\Sigma=\mathbb{O P}^{2}$. For convenience we encode each irreducible vector bundle $\mathcal{E}_{\lambda}$ on the Cayley plane $\mathbb{O P}^{2}$ by a weighted Dynkin diagram of type $E_{6}$, whose weights are the coordinates of $\lambda$ on the basis of fundamental weights (themselves in natural bijection with the nodes of the Dynkin diagram). For example we have

$$
\Omega_{\Sigma}^{1} \simeq \begin{gathered}
-21000 \\
0
\end{gathered}, \quad \Omega_{\Sigma}^{2} \simeq_{0}^{-30100} .
$$

The claim to be checked is that

$$
H^{14}\left(\Sigma, \Omega_{\Sigma}^{2}(-9)\right)=\mathbb{C}
$$

The root system, and the Weyl group $W$ of $E_{6}$ being rather complicated, we will use a different strategy than in the other case. If $\lambda=-3 \omega_{1}+\omega_{4}$ denotes the highest weight of $\Omega_{\Sigma}^{2}$, what we need to check is that $\mu=\lambda-9 \omega_{1}+\rho$ belongs to the $W$-orbit of $\rho$. Recall that $\rho$, the half sum of the positive roots, is also the sum of the fundamental weights. We can thus write

$$
\rho={ }^{11111}, \quad \mu=\stackrel{-B 1211}{1}
$$

where $B$ stands for eleven (and $A$ will stand for ten, below). Now we can let $W$ act through the simple reflections $s_{i}$ associated to the simple root $\alpha_{i}$. The recipe for the action of $s_{i}$ on a weight $\omega$, written in the basis of fundamental weights, is as follows: add the $i$-th coordinate to the $j$-th if $j$ is connected to $i$ in the Dynkin diagram; then change the $i$ th coordinate into 
its opposite. Here we start with a weight having a negative coordinate. Applying successively simple reflections corresponding to nodes with negative coordinates, we will end up with a dominant weight. Starting from $\mu$, the game goes as follows.

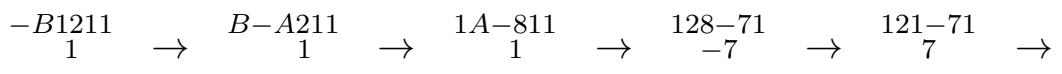

$$
\begin{aligned}
& \begin{array}{ccccc}
12-67-6 \\
7
\end{array} \rightarrow \begin{array}{c}
12-616 \\
7
\end{array} \rightarrow \begin{array}{c}
1-46-56 \\
1
\end{array} \rightarrow \begin{array}{c}
1-4151 \\
1
\end{array} \rightarrow \begin{array}{c}
-34-351 \\
1
\end{array} \rightarrow
\end{aligned}
$$

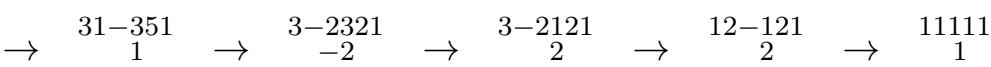

So we end up with $\rho$ after 14 operations. This indicates that we get a one dimensional cohomology group in degree fourteen. The claim is proved.

With the very same method we can prove that the one dimensional cohomology group whose existence is asserted by Lemma 4.1, is the only non-zero cohomology group of twisted form in a wide range. The precise statement is the following:

Lemma 5.1. Suppose that $p \leq c-1$ and $1 \leq k \leq r-p$. Then

$$
H^{q}\left(\Sigma, \Omega_{\Sigma}^{p}(-k)\right)=\delta_{q, r+2} \delta_{p, c-2} \delta_{k, r-c+1} \mathbb{C} .
$$

\section{REFERENCES}

[Be] Beauville A.: Determinantal hypersurfaces, Michigan Math. J. 48 (2000), 39-64.

[BB] Bialynicki-Birula A.: Some theorems on actions of algebraic groups, Annals of Math. 98 (1973), 480-497.

[Bo] Borcea C.: Smooth global complete intersections in certain compact homogeneous complex manifolds, J. Reine Angew. Math. 344 (1983), 65-70.

[CDP] Candelas P., Derrick E., Parkes L., Generalized Calabi-Yau Manifolds and the Mirror of a Rigid Manifold, Nucl.Phys. B 407 (1993), 115-154.

[Cy] Cynk S. Cohomologies of a double covering of a non-singular algebraic 3-fold, Math. Z. 240 (2002), 731-743.

[DM] Donagi R., Markman E.: Cubics, integrable systems, and Calabi-Yau threefolds, in Proceedings of the Hirzebruch 65 Conference on Algebraic Geometry, 199-221, Israel Math. Conf. Proc. 9, 1996.

[Fr] Freed D., Special Kähler manifolds, Comm. Math. Phys. 203 (1999), 31-52.

[GKZ] Gelfand I.M., Kapranov M.M., Zelevinsky A.V., Discriminants, resultants, and multidimensional determinants. Birkhäuser 1994.

[Gr] Griffiths, P.A., On the periods of certain rational integrals I, II, Annals of Math. 90 (1969), 460-495, 496-541.

[Ig] Igusa J., A classification of spinors up to dimension twelve, Amer. J. Math. 92 (1970), 997-1028.

[IK] Iliev A., Katzarkov L., On the Griffiths group of Fano manifolds of Calabi-Yau Hodge type, in preparation.

[IM1] Iliev A., Manivel L., The Chow ring of the Cayley plane, Compos. Math. 141 (2005), 146-160.

[IM2] Iliev A., Manivel L., On cubic hypersurfaces of dimension seven and eight, preprint 2011. 
[Ko] Kostant B., Lie algebra cohomology and generalized Schubert cells, Annals of Math. 77 (1963), 72-144.

[Ku1] Kuznetsov A., Derived categories of cubic and $V_{14}$ threefolds, Proc. Steklov Inst. Math. 246 (2004), 171-194.

[Ku2] Kuznetsov A., Homological projective duality, Publ. Math. Inst. Hautes tudes Sci. 105 (2007), 157-220.

[Ma] Manivel L., On the derived category of the Cayley plane, arXiv:math.AG/0907.2784, to appear in the Journal of Algebra.

[Na] Nagel J., The Abel-Jacobi map for complete intersections. Indag. Math. (N.S.) 8 (1997), no. 1, 95-113.

[Po] Popov V.L., Classification of the spinors of dimension fourteen, Uspehi Mat. Nauk 32 (1977), 199-200.

[Sch] Schimmrigk R.: Mirror Symmetry and String Vacua from a Special Class of Fano Varieties, Int. J. Mod. Phys. A 11 (1996), 3049-3096.

[Sn] Snow D.: Cohomology of twisted holomorphic forms on Grassmann manifolds and quadric hypersurfaces, Math. Ann. 276 (1986), 159-176.

[St] Steenbrink J.: Intersection form for quasi-homogeneous singularities, Compositio Math. 34 (1977), 211-223.

[Vo] Voisin C.: Symétrie Miroir, Panoramas et Synthèses 2, SMF 1996.

Department of Mathematics, Seoul National University, Seoul 151-747, KoREA

E-mail address: ailiev2001@yahoo.com

Institut Fourier, Université de Grenoble et CNRS, BP 74, 38402 SaintMartin d'Hères, France

E-mail address: Laurent.Manivel@ujf-grenoble.fr 